Supplementary Information for "Using deposition rate and substrate temperature to manipulate liquid crystal-like order in a vapor-deposited hexagonal columnar glass"

Authors: Camille Bishop*, ${ }^{1}$ Zhenxuan Chen, ${ }^{2}$ Michael F. Toney,${ }^{3}$ Harald Bock, ${ }^{4}$ Lian Yu, ${ }^{1,2}$

M.D. Ediger ${ }^{1}$

Corresponding Author email: camille.bishop@ nist.gov

1. University of Wisconsin - Madison, Department of Chemistry, 1101 University Ave, Madison, WI, 53706 (USA)

2. University of Wisconsin - Madison, School of Pharmacy, 777 Highland Ave, Madison, WI, 53705 (USA)

3. University of Colorado Boulder, Department of Chemical and Biological Engineering, Boulder, CO 80309 (USA)

4. Centre de Recherche Paul Pascal, CNRS \& Université de Bordeaux, 115, av. Schweitzer, 33600 Pessac (France)

Section 1. SGIWAXs calculation procedures.

To calculate $\mathrm{S}_{\text {GIWAXs, }}$, we first perform a background subtraction. All of the following procedures are shown for X-ray scattering from a glass deposited at $360 \mathrm{~K}$ at $0.4 \AA \mathrm{s}^{-1}$.

\title{
Background Subtraction
}

For all glasses, we find that the majority of the intensity from the $\mathrm{q} \sim 1.8 \AA^{-1}$ ( $\pi$-stacking) peak falls between $\mathrm{q}=1.65$ and $1.85 \AA^{-1}$. We therefore integrate this area (shown in black in Figure S1) at each value of the azimuthal angle $\chi$, with $\chi=0^{\circ}$ defined to be along $\mathrm{q}_{z}$. To obtain a low-q background, we perform the same integration on the region from $q=1.55$ to $1.65 \AA^{-1}$ (shown in orange on Figure S1). For the high-q background, the same is done for $q=1.85$ to $1.95 \AA^{-1}$, shown in red on Figure S1. 


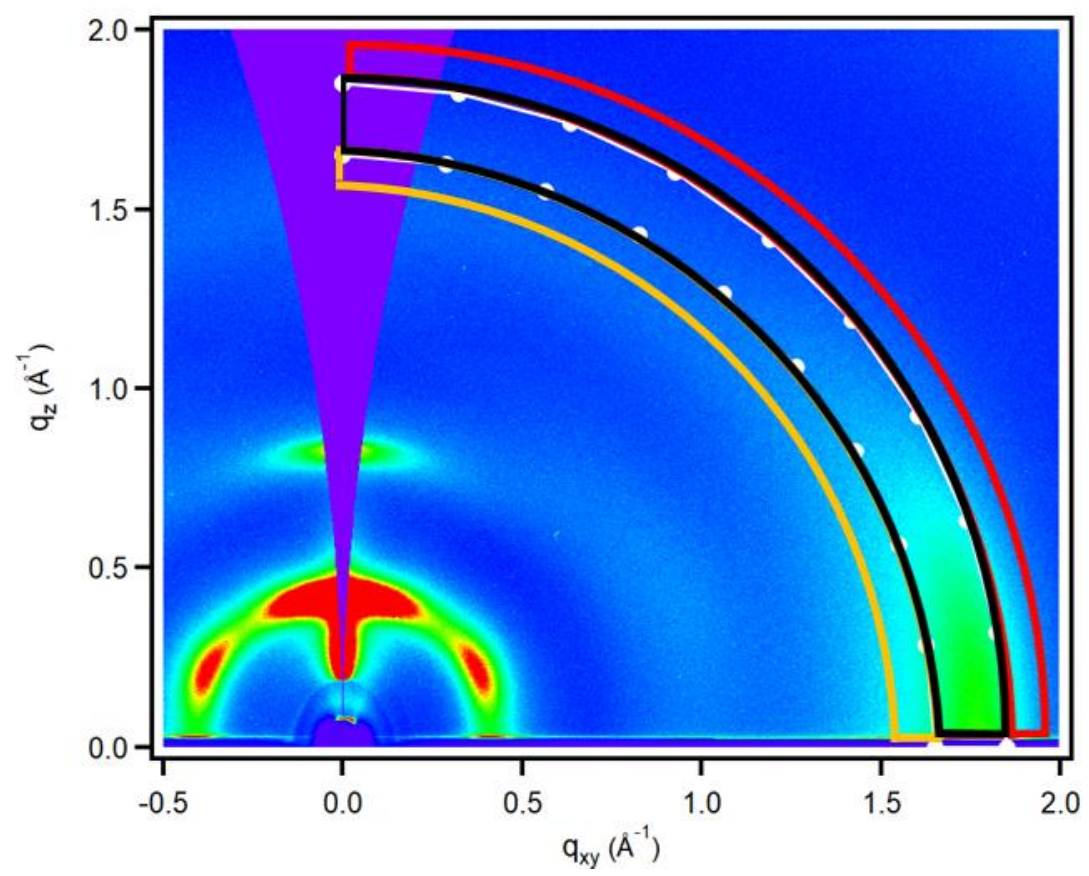

Figure S1. Integrated regions for $\pi$-stacking interaction (black), low-q background (orange), and high-q background (red).

These integrations result in the three curves seen in the upper part of Figure S2. To find the background subtracted curve at each value of $\chi$, we subtract the average of the low-q and high-q backgrounds as follows:

$$
I(\chi)=I_{q=1.65 \text { to } 1.85}(\chi)-\frac{I_{q=1.55 \text { to } 1.65}(\chi)+I_{q=1.85 \text { to } 1.95}(\chi)}{2}
$$

This results in the blue curve at the bottom of Figure S2.

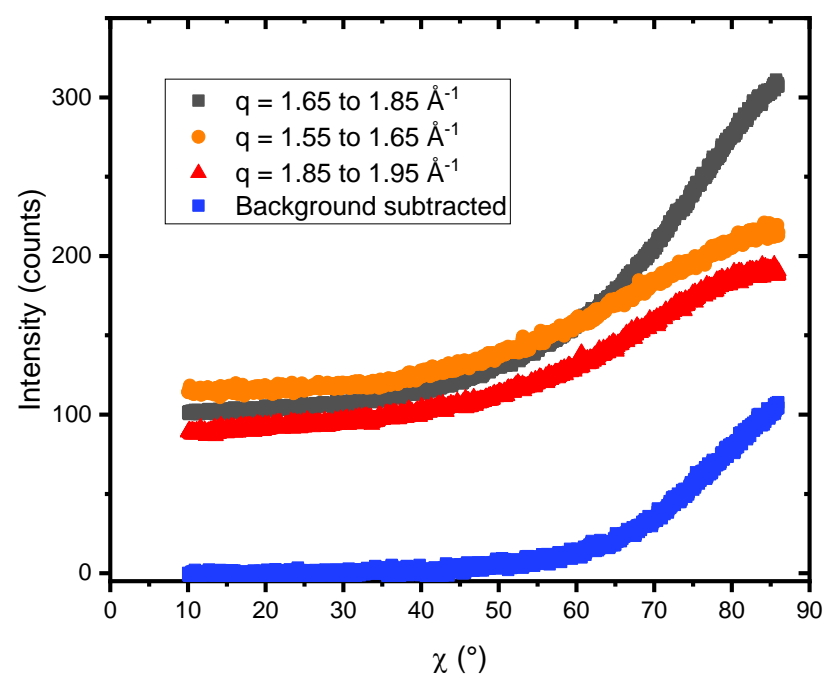


Figure S2. Raw integrations and background subtracted $\mathrm{I}(\chi)$ curves from Figure S1.

\section{Empirical extrapolation functions}

Due to the geometry of GIWAXS experiments, the data from $\chi=0^{\circ}$ to $\sim 10^{\circ}$ is inaccessible. Additionally, due to the edge of the sample surface, the data from $\chi \sim 86^{\circ}$ to $90^{\circ}$ is dominated by artefacts. For many samples, these regions have sufficient scattering that omission from the calculation of S SIWAXs will result in unphysical values. Therefore, we use empirical functions to extrapolate the scattering into the inaccessible regions, as shown in Figure S3.

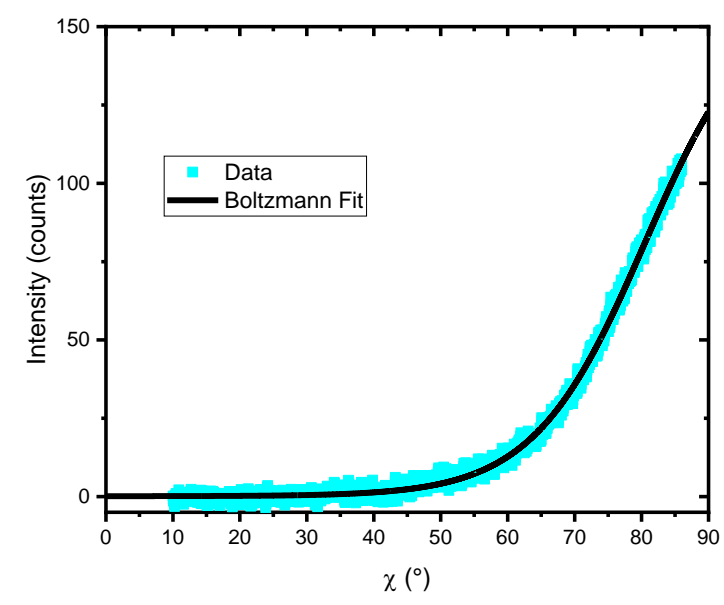

$$
I=A+\frac{B-A}{1+e^{(\chi-C) / D}}
$$

Figure S3. Background subtracted curve shown in Figure S2, with an empirical Boltzmann fit (equation shown to the right).

In the case of scattering that is extremely focused in-plane, the extrapolations may fail to capture the true scattering. Therefore, caution is required when interpreting results at extreme values of $\mathrm{S}_{\text {GIwAXS, }}$ such as those that do not fall on the linear fit in Figure $2 \mathrm{C}$ of the main text.

\section{Section 2. Determination of $d_{n n}$.}

\section{Selection of azimuthal range.}

To determine the average face-to-face nearest-neighbor distance, we integrate only in the region in which the scattering from this interaction is large. In this way, we find $\mathrm{d}_{\mathrm{nn}}$ for the dominant structural motif. We apply the background-subtracted curves as shown in SI Section 1 that are

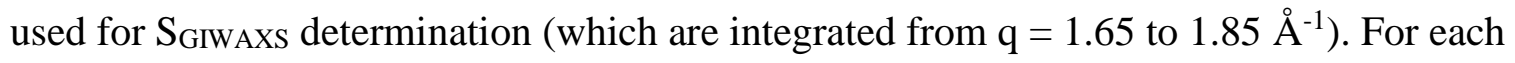
individual sample, we find the maximum scattering intensity. We then find the azimuthal angle $\chi$ 
at which the scattering intensity falls below $50 \%$ of the maximum, and define it as $\chi_{\min }$, the lower integration limit, shown schematically in Figure S4. For many samples, $\chi_{\max }$ occurs at the highest accessible $\chi$, as seen in Figure S4.

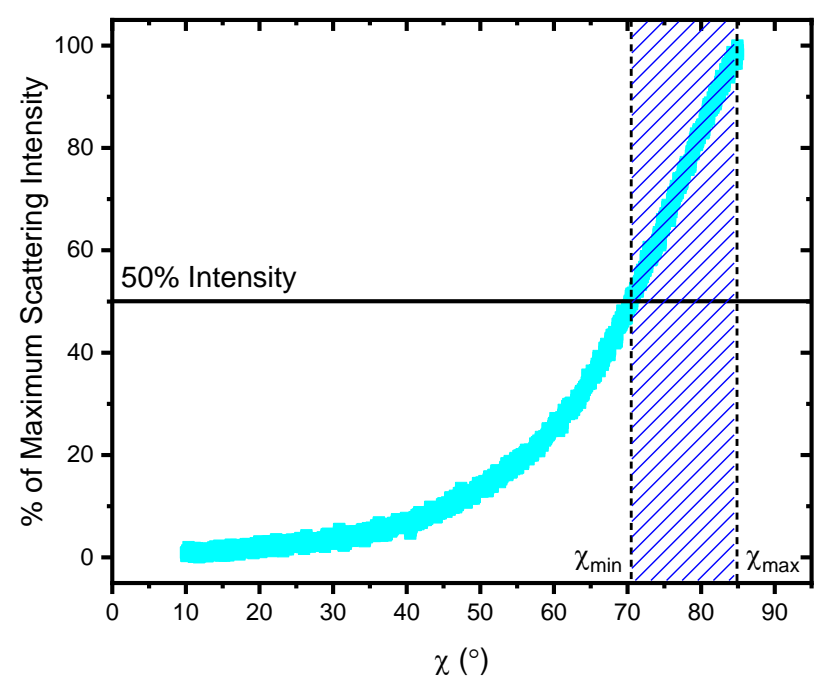

Figure S4. Determination of azimuthal integration limits for $\mathrm{d}_{\mathrm{nn}}$.

Determination of peak scattering position.

Next, the sample is integrated over all $\chi$ at each $\mathrm{q}$ in the azimuthal angle range determined by the previous step (in this case, from $\chi=71^{\circ}$ to $85^{\circ}$ ) to yield the curve seen in the top panel of Figure S5. The curve is smoothed using Savitzky-Golay smoothing with a $q=0.1 \AA^{-1}$ window, and then differentiated. We find that the peak position is independent of the smoothing window for smoothing windows of $\mathrm{q}=0.05 \AA^{-1}$ and higher, as shown in Figure S6. The peak position is determined by the zero of the first derivative:

$$
d_{n n}=\left.\frac{2 \pi}{q}\right|_{\frac{d I}{d q}=0}
$$




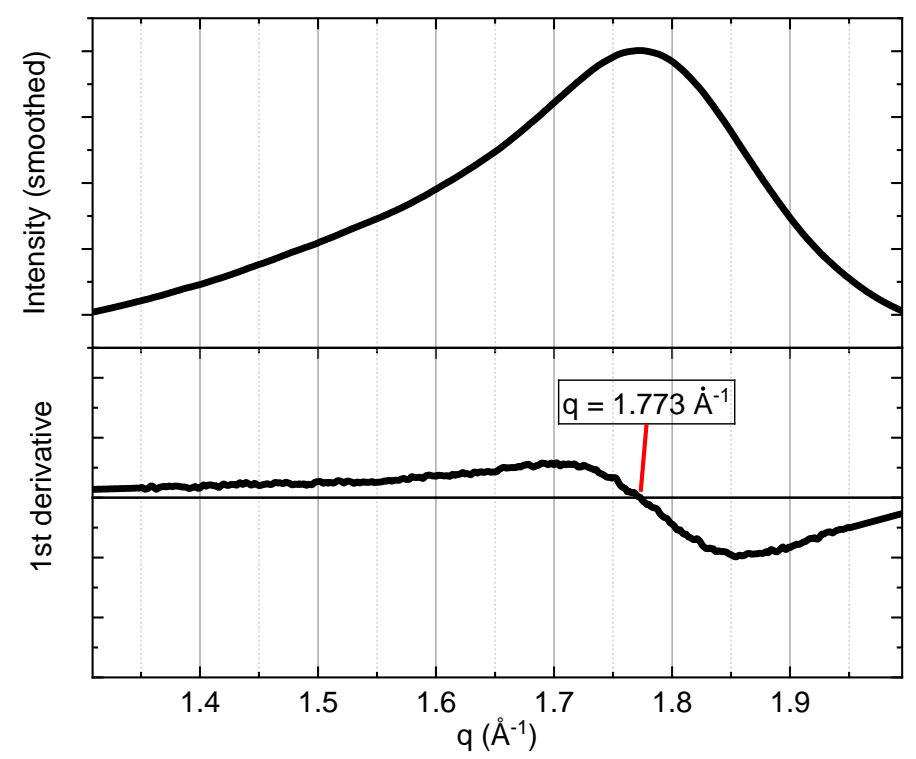

Figure S5. Determination of $\mathrm{d}_{\pi-\pi}$ from I vs. q curves.

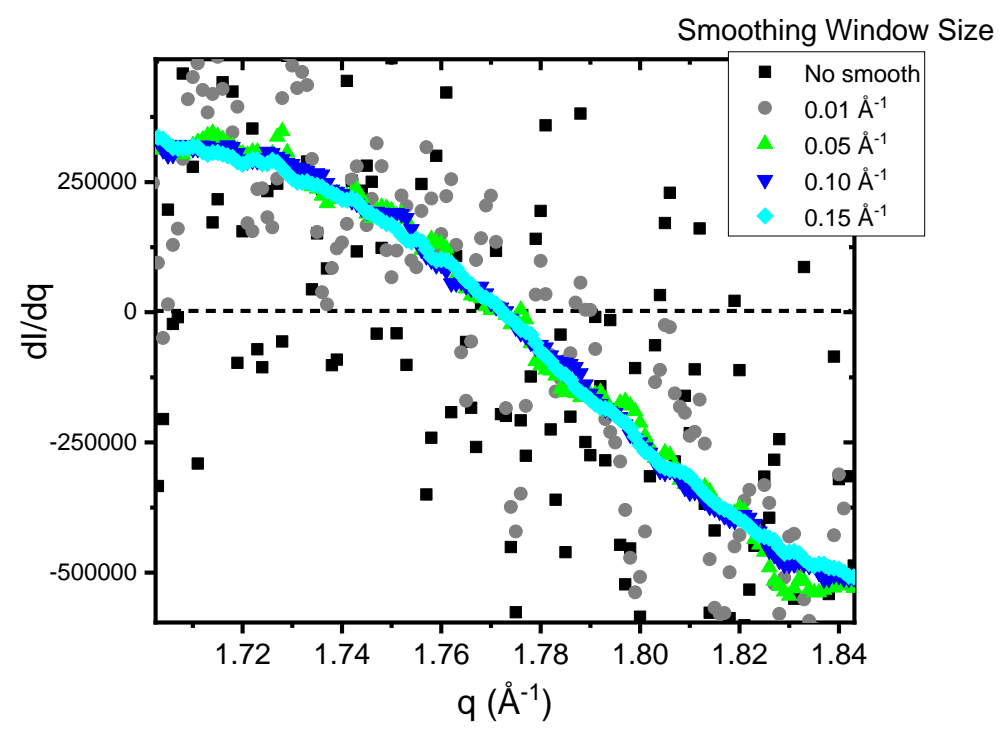

Figure S6. The first derivative used to determine $d_{n n}$ and its effect upon the resulting data. The qvalue of the zero of the first derivative, which is the peak position used to determine $\mathrm{d}_{\mathrm{nn}}$, is independent of smoothing window size above $0.05 \AA^{-1}$.

Section 3. Anisotropy of X-ray scattering and birefringence.

As addressed in the main text, at the deposition condition of $\mathrm{T}_{\text {sub }}=315 \mathrm{~K}$, deposition rate $=0.8$ $\AA \mathrm{s}^{-1}, \mathrm{~S}_{\text {GIWAXS }}=0$ while $\Delta \mathrm{n}>0$. The $\mathrm{x}$-ray scattering pattern for this sample is shown in Figure S6. The scattering of the $\mathrm{q} \sim 1.8 \AA^{-1}$ peak used to calculate $\mathrm{S}_{\text {GIWAXS }}$ is isotropic, and hence 
$\mathrm{S}_{\text {GIWAXS }}=0$. However, other features in the scattering pattern, such as the $\mathrm{q} \sim 0.4 \AA^{-1}$ peak are anisotropic. Given that the scattering pattern as a whole is anisotropic, there is no expectation that the birefringence should be precisely zero.

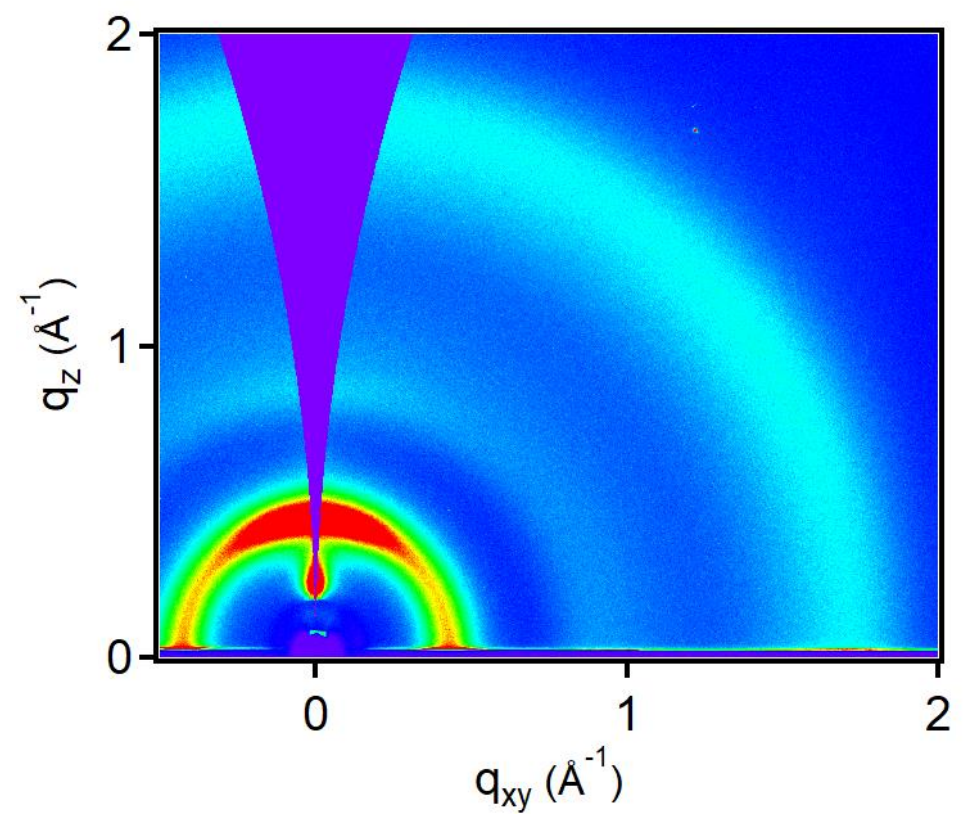

Figure S7. GIWAXS pattern of a glass deposited at $315 \mathrm{~K}, 10^{-0.1} \AA \mathrm{s}^{-1}$.

SI Section 4. Edge-to-edge disc spacing of molecules from $\mathrm{q} \sim 0.4 \AA^{-1}$ peak.

Analogously to the case for face-to-face disc packing seen in Figure 3, we analyzed the spacing for the hexagonal lattice. We first integrate over all $\chi=10^{\circ}$ to $85^{\circ}$ to plot I vs. $\mathrm{q}$ for all accessible scattering space. We then found $d$ for the edge-to-edge hexagonal packing by $d=2 \pi / q_{c}$, where $q_{c}$ is the center of a Pseudo-Voigt fit to the peak at $\mathrm{q} \sim 0.4 \AA^{-1}$. Results are shown in Figure S8. Figure $\mathrm{S} 9$ shows this data plotted against the effective deposition rate calculated using the "shift factor" of $9 \mathrm{~K} /$ decade found for hexagonal order in the main text. 


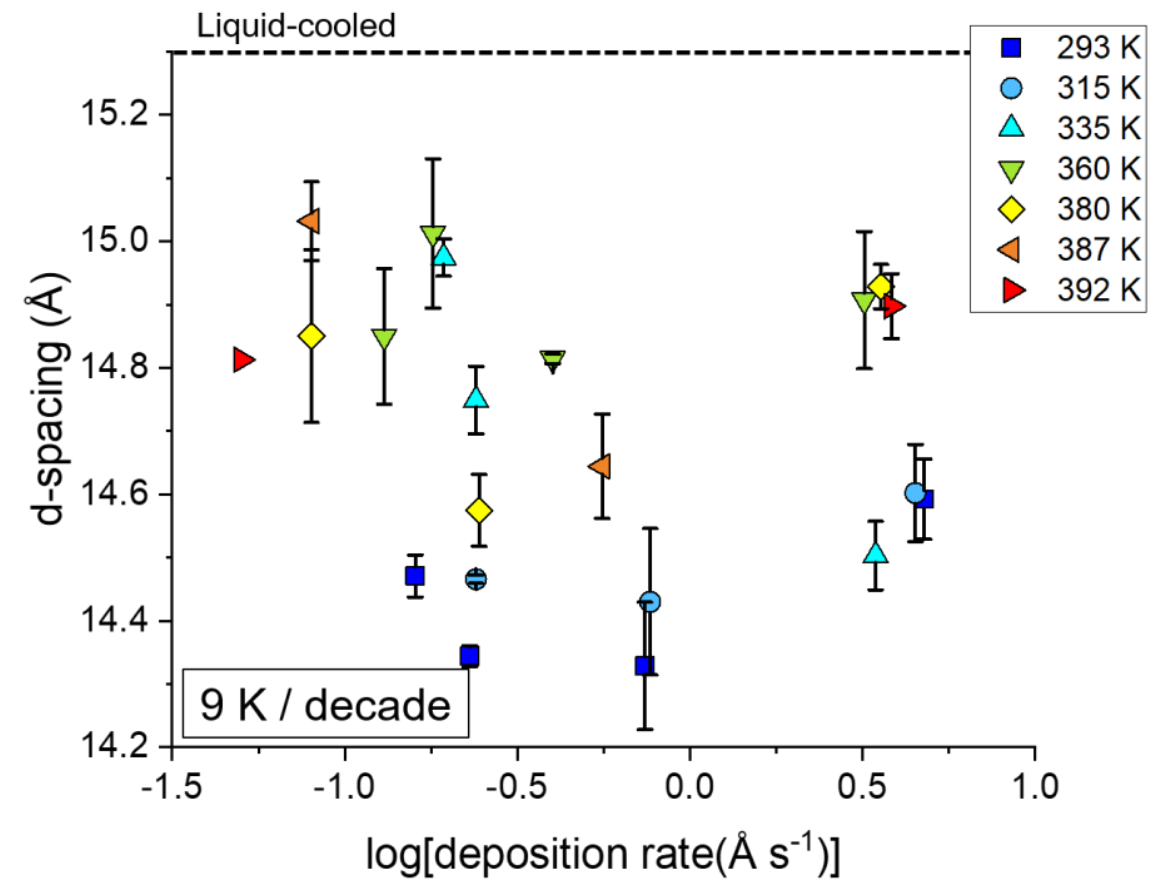

Figure S8. d-spacing of the hexagonal lattice peak found at $\mathrm{q} \sim 0.4 \AA^{-1}$ as a function of the log of the deposition rate.

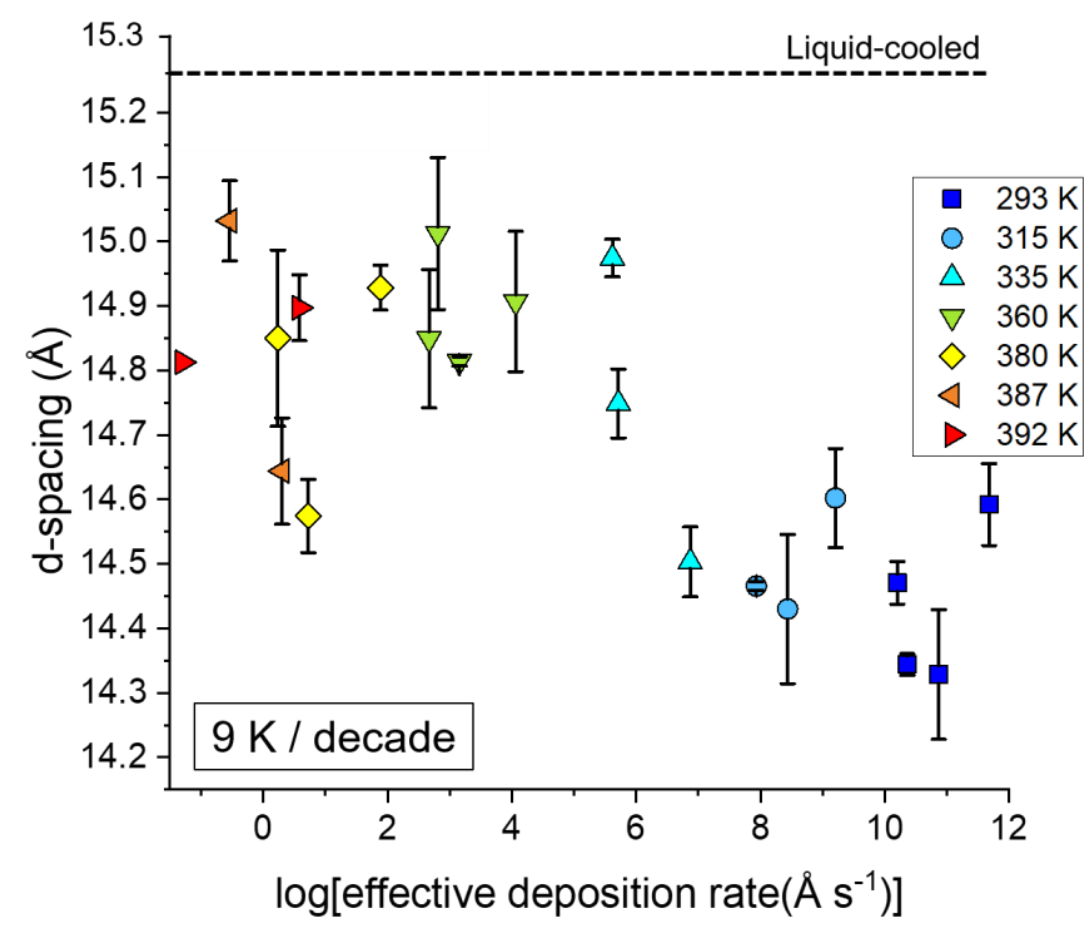

Figure S9. d-spacing of the hexagonal peak as a function of the effective deposition rate. 